Article

\title{
Supersymmetry of Relativistic Hamiltonians for Arbitrary Spin
}

\author{
Georg Junker 1,2 (D) \\ 1 European Southern Observatory, Karl-Schwarzschild-Straße 2, D-85748 Garching, Germany; \\ gjunker@eso.org \\ 2 Institut für Theoretische Physik I, Universität Erlangen-Nürnberg, Staudtstraße 7, \\ D-91058 Erlangen, Germany
}

Received: 16 August 2020; Accepted: 22 September 2020; Published: 24 September 2020 updates

\begin{abstract}
Hamiltonians describing the relativistic quantum dynamics of a particle with an arbitrary but fixed spin are shown to exhibit a supersymmetric structure when the even and odd elements of the Hamiltonian commute. Here, the supercharges transform between energy eigenstates of positive and negative energy. For such supersymmetric Hamiltonians, an exact Foldy-Wouthuysen transformation exists which brings it into a block-diagonal form separating the positive and negative energy subspaces. The relativistic dynamics of a charged particle in a magnetic field are considered for the case of a scalar (spin-zero) boson obeying the Klein-Gordon equation, a Dirac (spin one-half) fermion and a vector (spin-one) boson characterised by the Proca equation. In the latter case, supersymmetry implies for the Landé g-factor $g=2$.
\end{abstract}

Keywords: relativistic wave equation; Klein-Gordon equation; Dirac equation; Proca equation; supersymmetry

\section{Introduction}

Soon after the formulation of non-relativistic quantum mechanics by Heisenberg, Born, Jordan and Schrödinger in 1925 and 1926, Klein [1] and Gordon [2] made first attempts to develop a relativistic quantum wave formalism. This Klein-Gordon equation is known to have certain deficits for its quantum mechanical interpretation but nowadays is well accepted as being the correct quantum wave formalism for spin-zero particles. To overcome the problems of the Klein-Gordon equation, Dirac in 1928 [3,4] made an ansatz for a wave equation being linear in the time derivative and thus found his famous equation describing the relativistic quantum dynamics of spin one-half fermions. The relativistic spin-one equation, also known as Proca equation, has been developed by Proca [5] in 1936. In the same year Dirac [6] and later Fierz and Pauli [7,8] investigated relativistic wave equations for arbitrary spin. See also the later work by Bhabha [9]. A group theoretical discussion of such wave equations was then given by Bargmann and Wigner in 1948 [10].

In their fundamental work in 1950, Foldy and Wouthuysen [11] constructed a unitary transformation which separates the positive and negative energy states; that is, the Dirac Hamiltonian became block-diagonal. This work has triggered the study of the Hamiltonian form for the other wave equations. For example, Foldy [12] investigated the Klein-Gordon equation and Feshbach and Villars [13] made a unified approach to a Hamiltonian form of the Klein-Gordon and Dirac equation. The Hamiltonian form for the Proca equation has been studied by various authors including Duffin [14], Kemmer [15], Yukawa, Sakata and Taketani [16,17], Corben and Schwinger [18], as well as by Schrödinger [19]. The problem of restoring a block-diagonal Hamiltonian via a so-called exact Foldy-Wouthuysen (FW) transformation for a particle with an arbitrary spin is still attracting 
many researchers. See, for example, the recent work by Silenko [20] and Simulik [21] and the references therein.

The aim of the present work was to investigate another aspect of such relativistic Hamiltonians related to an underlying supersymmetric structure. Here, it shall be emphasised that this kind of supersymmetry is not related to the supersymmetry (SUSY) known from quantum field theory where the supercharges transform a bosonic state into a fermionic state and vice versa. Here, SUSY is to be understood in the context of supersymmetric quantum mechanics, where the supercharges transform between states of positive and negative Witten parity. Despite the fact that supersymmetric quantum mechanics was originally introduced by Nicolai [22] as the $(0+1)$-dimensional limit of SUSY quantum field theories, it is rather independent of the latter. Supersymmetric quantum mechanics became rather popular with the model introduced by Witten [23], being in essence a one-dimensional non-relativistic quantum system, which still finds many application in various areas of physics [24,25]. The first extension of supersymmetric quantum mechanics to the relativistic Dirac Hamiltonian is due to Jackiw [26] and Ui [27] and has found many applications, for example, in the analysis of the electronic properties of topological superconductors and graphene [28].

The main purpose of the present work was to show that such supersymmetric structure may also be established for other relativistic systems going beyond that of the Dirac Hamiltonians. That is, we will show under the requirement that the odd and even part of a relativistic Hamiltonian commute with each other it is possible to establish a SUSY structure similar to what is know in the Dirac case. To the best of our knowledge, such an extension for the Klein-Gordon case was only briefly discussed by Thaller in Section 5.5.3 of his book [29] and more explicit by Znojil [30]. Here, we will present a general approach of supersymmetric quantum mechanics for relativistic Hamiltonians for arbitrary but fixed spin. The explicit discussion will be limited to the scalar or spin-zero case, the Dirac case for spin- $\frac{1}{2}$ and the vector boson case, i.e., spin-one. In all three cases we consider the well-known problem of a charged particle in the presence of a magnetic field but now from the point of view of supersymmetric quantum mechanics. It turns out that all three models, in essence, are closely related to their non-relativistic counterparts in essentially the same way. In addition, SUSY requires for vector bosons a Landé g-factor $g=2$.

The paper is organised as follows. In the next section, the basic structure of relativistic Hamiltonians for an arbitrary spin are recalled. In Section 3, we then show that whenever the odd part commutes with the even part of such a Hamiltonian it is possible to construct an $N=2$ SUSY structure very similar to what is known for supersymmetric Dirac Hamiltonians [29,31]. It is also recalled that there exists an exact FW transformation bringing the Hamiltonian into a block-diagonal form. In Section 4, we explicitly discuss the cases of a charged spin-zero, spin one-half and spin-one particle in an external magnetic field. In all three cases, which cover all the currently known charged elementary particles, we find that the eigenvalue problem of the relativistic Hamiltonian can indeed be reduced to that of a non-relativistic one. Section 5 discusses the resolvent of supersymmetric relativistic Hamiltonians and again shows that for the three cases under consideration the Green's function, in essence, may be reduced to that of the associated non-relativistic Hamiltonian. Finally, in Section 6, we present a short conclusion and an outlook for possible further investigations and in the Appendix A we collect some useful relations for the spin $s=1$ case which are not that commonly known.

\section{Relativistic Hamiltonians for Arbitrary Spin}

In the Hamiltonian form of relativistic quantum mechanics, one puts the wave equation into a Schrödinger-like form

$$
\mathrm{i} \hbar \partial_{t} \Psi=H \Psi .
$$

The Hamiltonian in the above equation in general is of the form

$$
H=\beta m+\mathcal{E}+\mathcal{O}
$$


where $\beta^{2}=1$ acts as a grading operator and $m$ standard for the particle's mass. In addition to the mass term $\beta m$, the operator $\mathcal{E}$ represents the remaining even part of the Hamiltonian; that is, it commutes with the grading operator, $[\beta, \mathcal{E}]=0$. The operator $\mathcal{O}$ denotes the odd part of $H$ and obeys the anticommutation relation $\{\beta, \mathcal{O}\}=0$. For a particle with spin $s, s=0, \frac{1}{2}, 1, \frac{3}{2}, \ldots$, the Hilbert space $\mathcal{H}$ on which $H$ acts is given by

$$
\mathcal{H}=L^{2}\left(\mathbb{R}^{3}\right) \otimes \mathbb{C}^{2(2 s+1)},
$$

that is, the wave function $\Psi$ in (1) is a spinor with $2(2 s+1)$ components [20]. Let us note that we can decompose the Hilbert space $\mathcal{H}$ into a direct sum of the two eigenspaces of the grading operator $\beta$ with eigenvalue +1 and -1 , respectively,

$$
\mathcal{H}=\mathcal{H}_{+} \oplus \mathcal{H}_{-}, \quad \mathcal{H}_{ \pm}:=L^{2}\left(\mathbb{R}^{3}\right) \otimes \mathbb{C}^{2 s+1} .
$$

Obviously, $\mathcal{H}_{ \pm}$are simultaneously the subspaces where eigenvalues of $H$ are positive and negative, respectively.

For simplicity, let us put the relativistic Hamiltonian (2) into the form

$$
H=\beta \mathcal{M}+\mathcal{O},
$$

where we have absorbed the mass $m$ in the even mass operator $\mathcal{M}:=m+\beta \mathcal{E}$ with $[\beta, \mathcal{M}]=0$. Let us note here that above Hamiltonian is self-adjoint, i.e., $H=H^{\dagger}$, only for the case of fermions where $s=\frac{1}{2}, \frac{3}{2}, \ldots$ is a half-odd integer. For bosons, where $s$ takes integer values, the Hamiltonian is pseudo-hermitian, that is, $H=\beta H^{+} \beta$.

Choosing a representation where $\beta$ takes the diagonal form

$$
\beta=\left(\begin{array}{cc}
1 & 0 \\
0 & -1
\end{array}\right)
$$

where in the above the 1 denotes a $(2 s+1)$-dimensional unit matrix. The even and odd operators obeying $[\beta, \mathcal{M}]=0$ and $\{\beta, \mathcal{O}\}=0$ are necessarily of the form

$$
\mathcal{M}=\left(\begin{array}{cc}
M_{+} & 0 \\
0 & M_{-}
\end{array}\right), \quad \mathcal{O}=\left(\begin{array}{cc}
0 & A \\
(-1)^{2 s+1} A^{\dagger} & 0
\end{array}\right),
$$

where $M_{ \pm}: \mathcal{H}_{ \pm} \mapsto \mathcal{H}_{ \pm}$with $M_{ \pm}^{\dagger}=M_{ \pm}$is an operator mapping positive and negative energy states into positive and negative energy states, respectively. Whereas $A: \mathcal{H}_{-} \mapsto \mathcal{H}_{+}$maps a negative energy state into a positive energy state and $A^{+}: \mathcal{H}_{+} \mapsto \mathcal{H}_{-}$vice versa. With the above representation, the general relativistic spin-s Hamiltonian then takes the form

$$
H=\left(\begin{array}{cc}
M_{+} & A \\
(-1)^{2 s+1} A^{\dagger} & -M_{-}
\end{array}\right) .
$$

In the following section, we will show that under the assumption that the even and odd parts of the Hamiltonian (8) commute, i.e., $[\mathcal{M}, \mathcal{O}]=0$, it is possible to establish an $N=2$ supersymmetric structure being well-studied in supersymmetric quantum mechanics. This condition, which we will call the SUSY condition, also allows for an exact Foldy-Wouthuysen transformation.

\section{Supersymmetric Relativistic Hamiltonians for Arbitrary Spin}

As stipulated above, let us assume that the even mass operator $\mathcal{M}$ and the odd operator $\mathcal{O}$ commute. This SUSY condition implies that

$$
M_{+} A=A M_{-}, \quad A^{\dagger} M_{+}=M_{-} A^{\dagger} .
$$


As a consequence of this, the squared Hamiltonian (8) becomes block diagonal

$$
H^{2}=\left(\begin{array}{cc}
M_{+}^{2}+(-1)^{2 s+1} A A^{+} & 0 \\
0 & M_{-}^{2}+(-1)^{2 s+1} A^{\dagger} A
\end{array}\right) .
$$

Inspired by the construction of a SUSY structure for supersymmetric Dirac Hamiltonians [31], let us introduce the following SUSY Hamiltonian

$$
H_{\text {SUSY }}:=\frac{(-1)^{2 s+1}}{2 m c^{2}}\left(H^{2}-\mathcal{M}^{2}\right)=\frac{1}{2 m c^{2}}\left(\begin{array}{cc}
A A^{\dagger} & 0 \\
0 & A^{\dagger} A
\end{array}\right) \geq 0
$$

and the complex supercharges

$$
Q:=\frac{1}{\sqrt{2 m c^{2}}}\left(\begin{array}{cc}
0 & A \\
0 & 0
\end{array}\right), \quad Q^{+}=\frac{1}{\sqrt{2 m c^{2}}}\left(\begin{array}{cc}
0 & 0 \\
A^{+} & 0
\end{array}\right) .
$$

Here, $m>0$ is an arbitrary mass-like parameter, representing, for example, the mass of the relativistic particle in (2). It is obvious that these operators generate a transformation between positive and negative energy states. A straightforward calculation shows that these operators together with the Witten parity operator $W:=\beta$ form an $N=2$ SUSY system; that is,

$$
H_{\text {SUSY }}=\left\{Q, Q^{\dagger}\right\}, \quad\{Q, W\}=0, \quad Q^{2}=0=\left(Q^{\dagger}\right)^{2}, \quad\left[W, H_{\text {SUSY }}\right]=0, \quad W^{2}=1 .
$$

Let us note that $\mathcal{M}$ under condition (9) commutes with all operators of above algebra and thus constitutes a centre of the SUSY algebra (13). Hence, a relativistic arbitrary-spin Hamiltonian (8) obeying the SUSY condition (9) may be called a supersymmetric relativistic arbitrary-spin Hamiltonian.

Let us also note that for a supersymmetric relativistic arbitrary-spin Hamiltonian, there exists an exact Foldy-Wouthuysen transformation $U$ which brings (8) into the block-diagonal form [20]

$$
\begin{aligned}
H_{\mathrm{FW}} & :=U H U^{+}=\beta \sqrt{H^{2}} \\
& =\left(\begin{array}{cc}
\sqrt{M_{+}^{2}+(-1)^{2 s+1} 2 m c^{2} H_{+}} & 0 \\
0 & -\sqrt{M_{-}^{2}+(-1)^{2 s+1} 2 m c^{2} H_{-}}
\end{array}\right),
\end{aligned}
$$

where the partner Hamiltonians $H_{ \pm}$are defined by

$$
H_{+}:=\frac{1}{2 m c^{2}} A A^{\dagger}, \quad H_{-}:=\frac{1}{2 m c^{2}} A^{\dagger} A .
$$

In fact, it is known that under condition $[\mathcal{M}, \mathcal{O}]=0$, the exact Foldy-Wouthuysen transformation is explicitly given by $[20,32]$

$$
U:=\frac{|H|+\beta H}{\sqrt{2 H^{2}+2 \mathcal{M}|H|}}=\frac{1+\beta \operatorname{sgn} H}{\sqrt{2+\{\operatorname{sgn} H, \beta\}}}, \quad \operatorname{sgn} H:=\frac{H}{\sqrt{H^{2}}} .
$$

As a side remark, let us mention that the four projections operators

$$
P^{ \pm}:=\frac{1}{2}[1 \pm W], \quad \Lambda^{ \pm}:=\frac{1}{2}[1 \pm \operatorname{sgn} H],
$$

projecting onto the subspaces of positive/negative Witten parity and positive/negative eigenvalues of $H$, respectively, are related to each other via the same unitary transformation as $H$ and $H_{\mathrm{FW}}$

$$
P^{ \pm}=U \Lambda^{ \pm} U^{\dagger} .
$$


That is, the positive and negative energy eigenspaces are transformed via $U$ into spaces of positive and negative Witten parity. In fact, one may verify that $U$ may be represented in terms of these projection operators as follows

$$
U=\frac{P^{+} \Lambda^{+}+P^{-} \Lambda^{-}}{\sqrt{\left(P^{+} \Lambda^{+}+P^{-} \Lambda^{-}\right)\left(\Lambda^{+} P^{+}+\Lambda^{-} P^{-}\right)}} .
$$

The non-negative partner Hamiltonians $H_{ \pm} \geq 0$ are essential isospectral which means that their strictly positive eigenvalues are identical. The corresponding eigenstates are related to each other via a SUSY transformation. To be more explicit, let us assume these are given by

$$
H_{ \pm} \phi_{\varepsilon}^{ \pm}=\varepsilon \phi_{\varepsilon}^{ \pm}, \quad \phi_{\varepsilon}^{ \pm} \in \mathcal{H}_{ \pm}, \quad \varepsilon>0,
$$

then the SUSY transformation reads [31]

$$
\phi_{\varepsilon}^{+}=\frac{1}{\sqrt{2 m c^{2} \varepsilon}} A \phi_{\varepsilon}^{-}, \quad \phi_{\varepsilon}^{-}=\frac{1}{\sqrt{2 m c^{2} \varepsilon}} A^{\dagger} \phi_{\varepsilon}^{+} .
$$

Note that the energy eigenvalue $\varepsilon$ may be degenerate and above relations are valid for each of these energy eigenstates. We omit an additional index in $\phi_{\varepsilon}^{ \pm}$enumerating such a degeneracy. In addition, both partner Hamiltonians $H_{ \pm}$may have a non-trivial kernel; that is, there may exist one or several eigenstates with

$$
H_{ \pm} \phi_{0}^{ \pm}=0 .
$$

In this case, SUSY is said to be unbroken [31]. For these ground states, again we omit the index for a possible degeneracy. There exists no SUSY transformation relating $\phi_{0}^{+}$and $\phi_{0}^{-}$. The breaking of SUSY can be studied via the so-called Witten index $\Delta$ [33], which in the current context is identical to the Fredholm index of $A$, if it is a Fredholm operator, that is,

$$
\Delta \equiv \text { ind } A:=\operatorname{dim} \operatorname{ker} A-\operatorname{dim} \operatorname{ker} A^{\dagger}=\operatorname{dim} \operatorname{ker} H_{-}-\operatorname{dim} \operatorname{ker} H_{+} .
$$

Obviously a non-vanishing Witten index indicates that SUSY is unbroken. In connection with [29]

$$
\operatorname{dim} \operatorname{ker}\left(Q+Q^{\dagger}\right)=\operatorname{dim} \operatorname{ker} A+\operatorname{dim} \operatorname{ker} A^{\dagger}=\operatorname{dim} \operatorname{ker} H_{-}+\operatorname{dim} \operatorname{ker} H_{+}
$$

the kernels of $H_{ \pm}$; that is, the number of zero-energy states of $H_{ \pm}$are known. In general, however, the operator $A$ is not Fredholm and hence some regularized indices are studied [29,31].

Due to the SUSY condition (9), the mass operators commute with the associated partner Hamiltonians, $\left[M_{ \pm}, H_{ \pm}\right]=0$, and therefore have an identical set of eigenstates. Let us denote the corresponding eigenvalues of $M_{ \pm}$by $m_{ \pm} c^{2}$; that is,

$$
M_{ \pm} \phi_{\varepsilon}^{ \pm}=m_{ \pm} c^{2} \phi_{\varepsilon}^{ \pm}, \quad \varepsilon \geq 0,
$$

then obviously the eigenvalues and eigenstates of (14),

$$
H_{\mathrm{FW}} \psi_{\varepsilon}^{ \pm}=E_{ \pm} \psi_{\varepsilon}^{ \pm}
$$

are given by

$$
E_{ \pm}= \pm \sqrt{m_{ \pm}^{2} c^{4}+(-1)^{2 s+1} 2 m c^{2} \varepsilon}, \quad \psi_{\varepsilon}^{+}=\left(\begin{array}{c}
\phi_{\varepsilon}^{+} \\
0
\end{array}\right), \quad \psi_{\varepsilon}^{-}=\left(\begin{array}{c}
0 \\
\phi_{\varepsilon}^{-}
\end{array}\right) .
$$

Here, let us note that the mass eigenvalues may depend on the energy eigenvalues, $m_{ \pm}=m_{ \pm}(\varepsilon)$. Using the relation (9) in combination with the SUSY relation (21) and the above eigenvalue 
Equation (25), one may verify that $m_{+}(\varepsilon)=m_{-}(\varepsilon)$ for all $\varepsilon>0$. In essence, this means that the spectrum of a supersymmetric relativistic Hamiltonian is symmetric about zero with a possible exception at $\pm m_{ \pm}(0) c^{2}$; that is, if $\varepsilon=0$, which may only occur in the case of unbroken SUSY.

The eigenstates of the original Hamiltonian (8) are then easily found via the unitary transformation $\Psi_{\varepsilon}^{ \pm}=U^{\dagger} \psi_{\varepsilon}^{ \pm}$having the same eigenvalues $E_{ \pm}$given above. Hence, the eigenvalue problem of a supersymmetric relativistic spin-s Hamiltonian can be reduced to the simultaneous eigenvalue problems for $M_{ \pm}$and $H_{ \pm}$on $\mathcal{H}_{ \pm}$.

It will turn out in the examples to be discussed below that the partner Hamiltonians $H_{ \pm}$and the mass operators $M_{ \pm}$are in essence represented by a non-relativistic Schrödinger-like Hamiltonian $H_{\mathrm{NR}}$ and/or some constant operator. To be more precise, we will show for all three cases- $s=0, \frac{1}{2}$ and 1 -discussed below that the FW-transformed relativistic Hamiltonian takes the form

$$
H_{\mathrm{FW}}=\beta m c^{2} \sqrt{1+\frac{2 H_{\mathrm{NR}}}{m c^{2}}}
$$

with $H_{\mathrm{NR}}$ representing the associate non-relativistic Hamiltonian as

$$
\lim _{c \rightarrow \infty}\left(P^{ \pm} H_{\mathrm{FW}} P^{ \pm} \mp m c^{2}\right)= \pm \lim _{c \rightarrow \infty}\left(m c^{2} \sqrt{1+2 H_{\mathrm{NR}} / m c^{2}}-m c^{2}\right)= \pm H_{\mathrm{NR}} .
$$

\section{Examples}

In the following subsections we will consider a relativistic charged particle with charge $e$ and mass $m$ in an external magnetic field $\vec{B}:=\vec{\nabla} \times \vec{A}$ characterised by a vector potential $\vec{A}$. The symbol $\vec{\pi}$ stands for the kinetic momentum; that is, $\vec{\pi}:=\vec{p}-e \vec{A} / c$, where $c$ denotes the speed of light. We will consider the case of a scalar particle with spin $s=0$, a Dirac particle with $s=\frac{1}{2}$ and a vector boson having $\operatorname{spin} s=1$.

\subsection{The Klein-Gordon Hamiltonian with Magnetic Field}

The Schrödinger form of the Klein-Gordon equation as been considered by Feshbach and Villars in [13] where they have shown that the Klein-Gordon equation for a charged particle in a magnetic field can be put into the Schrödinger form (1) where the pseudo-Hermitian Hamiltonian is given by

$$
H=\left(\begin{array}{cc}
m c^{2}+\frac{\vec{\pi}^{2}}{2 m} & \frac{\vec{\pi}^{2}}{2 m} \\
-\frac{\vec{\pi}^{2}}{2 m} & -m c^{2}-\frac{\vec{\pi}^{2}}{2 m}
\end{array}\right), \quad \mathcal{H}=L^{2}\left(\mathbb{R}^{3}\right) \otimes \mathbb{C}^{2} .
$$

Comparing this with the general form (8), we may identify the operators $M_{ \pm}$and $A$ as follows:

$$
M_{ \pm}=\frac{1}{2 m} \vec{\pi}^{2}+m c^{2}, \quad A=\frac{1}{2 m} \vec{\pi}^{2} .
$$

Obviously, these operators are identical up to an additional constant $M_{ \pm}=A+m c^{2}$ and hence the condition (9); that is, $\left[M_{ \pm}, A\right]=0$ is trivially fulfilled. In other words, the Klein-Gordon Hamiltonian (30) for a charged scalar particle in the presence of an arbitrary magnetic field represents a supersymmetric relativistic spin-zero Hamiltonian. Let us note that the two operators (31) in essence are given by the Landau Hamiltonian $H_{\mathrm{L}}:=(\vec{p}-e \vec{A} / c)^{2} / 2 m=\vec{\pi}^{2} / 2 m$ of a non-relativistic spinless charged particle of mass $m$ in a magnetic field; that is, $M_{ \pm}=H_{\mathrm{L}}+m c^{2}$ and $A=H_{\mathrm{L}}$. As a consequence, the eigenvalue problem for (30) is reduced to that of $H_{\mathrm{L}}$. The FW transformed Hamiltonian explicitly reads

$$
H_{\mathrm{FW}}=\left(\begin{array}{cc}
\sqrt{\left(H_{\mathrm{L}}+m c^{2}\right)^{2}-H_{\mathrm{L}}^{2}} & 0 \\
0 & -\sqrt{\left(H_{\mathrm{L}}+m c^{2}\right)^{2}-H_{\mathrm{L}}^{2}}
\end{array}\right)=\beta m c^{2} \sqrt{1+\frac{2 H_{\mathrm{L}}}{m c^{2}}} .
$$


Let us denote the eigenvalues of $H_{\mathrm{L}}$ by $\epsilon$, then the eigenvalues of $M_{ \pm}$and $H_{ \pm}=H_{\mathrm{L}}^{2} / 2 m c^{2}$ read

$$
m_{ \pm} c^{2}=m c^{2}+\epsilon, \quad \varepsilon=\frac{\epsilon^{2}}{2 m c^{2}}
$$

and via relation (27) we find the eigenvalues of the Klein-Gordon Hamilonian

$$
E_{ \pm}= \pm \sqrt{m_{ \pm}^{2} c^{4}-2 m c^{2} \varepsilon}= \pm \sqrt{\left(m c^{2}+\epsilon\right)^{2}-\epsilon^{2}}= \pm m c^{2} \sqrt{1+\frac{2 \epsilon}{m c^{2}}}
$$

which is a result expected from relation (32). For a non-vanishing constant magnetic field, say in the $z$-direction $\vec{B}=B \vec{e}_{z}$ with $B \neq 0$, the eigenvalues of $H_{\mathrm{L}}$ are the well-known Landau levels [34,35]

$$
\epsilon=\hbar \omega_{c}\left(n+\frac{1}{2}\right)+\frac{\hbar^{2} k_{z}^{2}}{2 m}, \quad n \in \mathbb{N}_{0}, \quad k_{z} \in \mathbb{R}, \quad \omega_{c}:=\frac{|e B|}{m c} .
$$

As $\epsilon \geq \hbar \omega_{c} / 2>0$ so is $\varepsilon>0$ and hence $\operatorname{dim} \operatorname{ker} H_{-}=\operatorname{dim} \operatorname{ker} H_{-}=0$. In other words, the Witten index (23) vanishes and SUSY is broken for the Klein-Gordon Hamiltonian in a constant magnetic field.

\subsection{The Dirac Hamiltonian with Magnetic Field}

The Dirac equation representing the relativistic dynamics of spin- $\frac{1}{2}$ fermions has intensively been studied since its introduction. See, for example, the excellent book by Thaller [29]. For a charged particle in an arbitrary external magnetic field, the Dirac Hamiltonian reads

$$
H=\left(\begin{array}{cc}
m c^{2} & c \vec{\sigma} \cdot \vec{\pi} \\
c \vec{\sigma} \cdot \vec{\pi} & -m c^{2}
\end{array}\right)
$$

where $\vec{\sigma}=\left(\sigma_{1}, \sigma_{2}, \sigma_{3}\right)^{T}$ stands for a three-dimensional vector who's components are given by the Pauli matrices acting on $\mathbb{C}^{2}$, thus representing the spin- $\frac{1}{2}$ degree of freedom. Comparing this with the general form (8), we may identify the operators $M_{ \pm}=m c^{2}$ and $A=c \sigma \cdot \vec{\pi}=A^{\dagger}$ and note that condition (9) is trivially fulfilled. Hence, the Dirac Hamiltonian (36) is indeed supersymmetric and its FW transformed form is known [32] to be expressible in terms of the non-relativistic Pauli Hamiltonian for a spin- $\frac{1}{2}$ particle with Landé $g$-factor $g=2$.

$$
H_{\mathrm{P}}:=\frac{1}{2 m}(\vec{\sigma} \cdot \vec{\pi})^{2}=\frac{1}{2 m}(\vec{p}-e \vec{A} / c)^{2}-\frac{e \hbar}{m c} \vec{B} \cdot \vec{\sigma} .
$$

Obviously, the partner Hamiltonians can be identified with the Pauli Hamiltonian $H_{ \pm}=A^{2} / 2 m c^{2}=H_{\mathrm{P}}$ and we find for the FW transformed Dirac Hamiltonian

$$
H_{\mathrm{FW}}=\left(\begin{array}{cc}
\sqrt{m^{2} c^{4}+2 m c^{2} H_{\mathrm{p}}} & 0 \\
0 & -\sqrt{m^{2} c^{4}+2 m c^{2} H_{\mathrm{p}}}
\end{array}\right)=\beta m c^{2} \sqrt{1+\frac{2 H_{\mathrm{P}}}{m c^{2}}} .
$$

As shown by Aharonov and Casher [36], for a magnetic field having only a $z$-component, $\vec{B}=B(x, y) \vec{e}_{z}$, the ground-state energy of $H_{\mathrm{P}}$ is zero and the Witten index (23) in essence is given by the flux $F=\int_{\mathbb{R}^{2}} \mathrm{~d} x \mathrm{~d} y B(x, y)$ measured in units of the magnetic flux quantum $\Phi_{0}:=2 \pi \hbar c /|e|$. Hence, SUSY is unbroken in this case. For details, see for example [31], where it is shown that the Pauli Hamiltonian for this magnetic field provides an additional SUSY structure within both subspaces $\mathcal{H}_{ \pm}$.

Finally, for an electron $(e<0)$ in a constant magnetic field, the eigenvalues of $H_{\mathrm{P}}$ are well-known

$$
\varepsilon=\hbar \omega_{c}\left(n+\frac{1}{2}+s_{z}\right)+\frac{\hbar^{2} k_{z}^{2}}{2 m}, \quad n \in \mathbb{N}_{0}, \quad k_{z} \in \mathbb{R}, \quad s_{z} \in\left\{-\frac{1}{2}, \frac{1}{2}\right\},
$$


and the eigenvalues for (36) determined via (27) are the relativistic Landau levels first obtained by Rabi in 1928 [37]

$$
E_{ \pm}= \pm \sqrt{m^{2} c^{2}+\hbar^{2} c^{2} k_{z}^{2}+2 m c^{2} \hbar \omega_{c}\left(n+1 / 2+s_{z}\right)} .
$$

The degeneracy for each set of quantum numbers $\left(n, s_{z}, k_{z}\right)$ is given by the largest integer which is strictly less than $|F| / \Phi_{0}$ and is only finite in case the magnetic field has a compact support.

\subsection{The Spin-1 Hamiltonian with Magnetic Field}

Initiated by Proca's work [5], several authors have studied relativistic spin-one wave equations. Let us mention here the work by Duffin [14], by Kemmer [15] and by Yukawa, Sakata and Taketani [16,17]. An early study of the eigenvalue problem is due to Corben and Schwinger [18]. A relativistic Hamiltonian was studied, for example, by Young and Bludman [38], by Krase et al. [39] and by Tsai and coworkers [40,41]. The later work by Daicic and Frankel [42] presents an alternative solution to eigenvalue problem of the spin-one Hamiltonian in an external magnetic field. A recent treatment via FW transformation can be found in ref. [43].

The Hamiltonian of a charged spin-one particle with a priori arbitrary $g$-factor is given by, see for example [38,42,43],

$$
H=\left(\begin{array}{cc}
m c^{2}+\frac{\vec{\pi}^{2}}{2 m}-\frac{g e \hbar}{2 m c}(\vec{S} \cdot \vec{B}) & \frac{\vec{\pi}^{2}}{2 m}-\frac{1}{m}(\vec{S} \cdot \vec{\pi})^{2}+\frac{(g-2) e \hbar}{2 m c}(\vec{S} \cdot \vec{B}) \\
-\frac{\vec{\pi}^{2}}{2 m}+\frac{1}{m}(\vec{S} \cdot \vec{\pi})^{2}-\frac{(g-2) e \hbar}{2 m c}(\vec{S} \cdot \vec{B}) & -m c^{2}-\frac{\vec{\pi}^{2}}{2 m}+\frac{g e \hbar}{2 m c}(\vec{S} \cdot \vec{B})
\end{array}\right),
$$

where $\vec{S}=\left(S_{1}, S_{2}, S_{3}\right)^{T}$ is a vector who's components are $3 \times 3$ matrices acting on $\mathbb{C}^{3}$ and obeying the $S O(3)$ algebra $\left[S_{i}, S_{j}\right]=\mathrm{i} \varepsilon_{i j k} S_{k}$ representing the spin-one-degree of freedom of the particle. Again, we may identify the operators

$$
M_{ \pm}:=m c^{2}+\frac{\vec{\pi}^{2}}{2 m}-\frac{g e \hbar}{2 m c}(\vec{S} \cdot \vec{B}), \quad A:=\frac{\vec{\pi}^{2}}{2 m}-\frac{1}{m}(\vec{S} \cdot \vec{\pi})^{2}+\frac{(g-2) e \hbar}{2 m c}(\vec{S} \cdot \vec{B})=A^{\dagger} .
$$

From now on, let us assume that the magnetic field $\vec{B}$ is constant, i.e., $\vec{A}=\frac{1}{2} \vec{B} \times \vec{r}$. Under this condition, one may verify that

$$
\left[M_{ \pm}, A\right]=(g-2) \frac{e \hbar}{2 m^{2} c}\left[(\vec{S} \cdot \vec{B}),(\vec{S} \cdot \vec{\pi})^{2}\right]
$$

Hence, the SUSY condition (9) is fulfilled if and only if $g=2$. In other words, the relativistic spin-one Hamiltonian (41) is a supersymmetric Hamiltonian if the gyromagnetic factor is given by $g=2$. For a detailed discussion, we refer to the recent paper [44]. Here, we remark that the "Vector Boson" Hamiltonian

$$
H_{\mathrm{V}}:=\frac{\vec{\pi}^{2}}{2 m}-\frac{e \hbar}{m c}(\vec{S} \cdot \vec{B})
$$

represents the non-relativistic Hamiltonian of a charged spin-one particle in a magnetic field with gyromagnetic factor $g=2$. Note that (44) is related to the quantity $\alpha$ introduced by Weaver [45] by $H_{V}=\frac{\alpha}{2 m}$. That this is indeed the non-relativistic version of (41) was already mentioned in ref. [39]. With this we have $M_{ \pm}=H_{\mathrm{V}}+m c^{2}$ and with relation (3.18) and (3.19) from ref. [42], see also the Appendix A, a straightforward calculation shows that $A^{2}=H_{\mathrm{V}}^{2}$. That is, the partner Hamiltonians read $H_{ \pm}=H_{\mathrm{V}}^{2} / 2 m c^{2}$ and the transformed FW Hamiltonian takes the form

$$
H_{\mathrm{FW}}=\left(\begin{array}{cc}
\sqrt{\left(H_{\mathrm{V}}+m c^{2}\right)^{2}-H_{\mathrm{V}}^{2}} & 0 \\
0 & -\sqrt{\left(H_{\mathrm{V}}+m c^{2}\right)^{2}-H_{\mathrm{V}}^{2}}
\end{array}\right)=\beta m c^{2} \sqrt{1+\frac{2 H_{\mathrm{V}}}{m c^{2}}}
$$


The eigenvalues of (44) are given by (we assume $e<0$ )

$$
\epsilon=\hbar \omega_{c}\left(n+\frac{1}{2}+s_{z}\right)+\frac{\hbar^{2} k_{z}^{2}}{2 m}, \quad n \in \mathbb{N}_{0}, \quad k_{z} \in \mathbb{R}, \quad s_{z} \in\{-1,0,1\} .
$$

Hence, the spectrum of the partner Hamiltonians $H_{ \pm}$is given by $\varepsilon=\epsilon^{2} / 2 m c^{2}$ and SUSY is unbroken as $\epsilon=0$ for $n=0, s_{z}=-1$ and $k_{z}= \pm 1 / \lambda_{L}$ with $\lambda_{L}:=\sqrt{\hbar / m \omega_{c}}=\sqrt{\hbar c /|e B|}$ being the Lamor length [42]. That is, SUSY is unbroken for a spin-1 particle in a homogeneous magnetic field but the Witten index remains zero as $H_{+}=H_{-}$and therefore $\operatorname{dim} \operatorname{ker} H_{+}=\operatorname{dim} \operatorname{ker} H_{-}$. The corresponding eigenvalues of (41) are given by

$$
E_{ \pm}= \pm \sqrt{m^{2} c^{2}+\hbar^{2} c^{2} k_{z}^{2}+2 m c^{2} \hbar \omega_{c}\left(n+1 / 2+s_{z}\right)}
$$

which is identical in form to the Dirac case (40) but $s_{z}$ now taking the integer values as given in (46). In fact, for $k_{z}=0, n=0$ and $s_{z}=-1$, the above eigenvalues would become complex if $|B|>m^{2} c^{3} /|e| \hbar$. Such large magnetic fields would imply $\lambda_{L}<\lambda_{C}:=\hbar / m c$; that is, the Lamor wavelength being smaller than the Compton wavelength of the vector boson. Note that confining a quantum particle to a region of the order of its Compton wavelength $\Delta x \sim \lambda_{C}$ implies by the uncertainty relation a momentum fluctuation $\Delta p \sim m c$ and thus a single particle description is no longer appropriate. In other words for such large magnetic fields a description via quantum field theory must be applied.

\section{The Resolvent of Supersymmetric Relativistic Arbitrary-Spin Hamiltonians}

In this section, we want to study the resolvent or Green's function of supersymmetric relativistic arbitrary-spin Hamiltonians defined as

$$
G(z):=\frac{1}{H-z}, \quad z \in \mathbb{C} \backslash \operatorname{spec} H .
$$

For this, is it convenient to first look at the iterated resolvent which is given by

$$
g(\zeta):=\frac{1}{H^{2}-\zeta}, \quad \zeta \in \mathbb{C} \backslash \operatorname{spec} H^{2}
$$

and is related with (48) via the obvious relation

$$
G(z)=(H+z) g\left(z^{2}\right)
$$

$H^{2}$ is block-diagonal and so is $g$; hence, it can be put into the form

$$
g(\zeta):=\left(\begin{array}{cc}
g^{+}(\zeta) & 0 \\
0 & g^{-}(\zeta)
\end{array}\right) \quad \text { with } \quad g^{ \pm}(\zeta):=\frac{1}{M_{ \pm}^{2}+(-1)^{2 s+1} 2 m c^{2} H_{ \pm}-\zeta}
$$

As a result, the resolvent (48) can be expressed in terms of (51) as follows:

$$
G(z)=\left(\begin{array}{cc}
\left(z+M_{+}\right) g^{+}\left(z^{2}\right) & A g^{-}\left(z^{2}\right) \\
(-1)^{2 s+1} A^{+} g^{+}\left(z^{2}\right) & \left(z-M_{-}\right) g^{-}\left(z^{2}\right)
\end{array}\right) .
$$

In the following subsections, we will explicitly consider the three cases discussed in the previous section. It will turn out that for these three cases, the diagonal elements $g^{ \pm}$of the iterated Green's 
function can be expressed in terms of the Green's function of the corresponding non-relativistic Hamiltonian $H_{\mathrm{NR}}$; that is,

$$
g^{ \pm}(\zeta)=\frac{1}{2 m c^{2}} G_{\mathrm{NR}}\left(\frac{\zeta}{2 m c^{2}}-\frac{m c^{2}}{2}\right), \quad G_{\mathrm{NR}}(\xi):=\frac{1}{H_{\mathrm{NR}}-\xi^{\prime}}, \quad \xi \in \mathbb{C} \backslash \operatorname{spec} H_{\mathrm{NR}}
$$

Note that the relation $\xi=z^{2} / 2 m c^{2}-m c^{2} / 2$, which can be put into the form $z= \pm m c^{2} \sqrt{1+2 \xi / m c^{2}}$, in essence reflects the relation (28).

\subsection{The Resolvent of the Klein-Gordon Hamiltonian with Magnetic Field}

Following the discussion of the first example of Section 4, we may express all relevant operators in terms of the Landau Hamiltonian $H_{\mathrm{L}}=\vec{\pi}^{2} / 2 m$. Explicitly, we have

$$
M_{ \pm}=H_{\mathrm{L}}+m c^{2}, \quad H_{ \pm}=H_{\mathrm{L}}^{2} / 2 m c^{2}, \quad A=H_{\mathrm{L}}{ }^{\dagger},
$$

which results in the iterated resolvents

$$
g^{ \pm}(\zeta):=\frac{1}{\left(H_{\mathrm{L}}+m c^{2}\right)^{2}-H_{\mathrm{L}}^{2}-\zeta}=\frac{1}{2 m c^{2}} G_{\mathrm{L}}\left(\frac{\zeta}{2 m c^{2}}-\frac{m c^{2}}{2}\right)
$$

where $G_{L}$ stands for the Green function of the Landau Hamiltonian in terms of which the Klein-Gordon Hamiltonian reads

$$
H=\left(\begin{array}{cc}
m c^{2}+H_{\mathrm{L}} & H_{\mathrm{L}} \\
-H_{\mathrm{L}} & -m c^{2}-H_{\mathrm{L}}
\end{array}\right) .
$$

The Green's function then reads in terms of the Landau Hamiltonian

$$
G(z)=\frac{1}{2 m c^{2}}\left(\begin{array}{cc}
z+m c^{2}+H_{\mathrm{L}} & H_{\mathrm{L}} \\
-H_{\mathrm{L}} & z-m c^{2}-H_{\mathrm{L}}
\end{array}\right) G_{\mathrm{L}}\left(\frac{z^{2}}{2 m c^{2}}-\frac{m c^{2}}{2}\right) .
$$

\subsection{The Resolvent of the Dirac Particle in a Magnetic Field}

As in the above discussion, let us first recall the observations made in Section 4.2; that is,

$$
M_{ \pm}=m c^{2}, \quad H_{ \pm}=A^{2} / 2 m c^{2}=H_{\mathrm{P}}, \quad A=c \vec{\sigma} \cdot \vec{\pi},
$$

which provide us with the components of the iterated kernel

$$
g^{ \pm}(\zeta):=\frac{1}{2 m c^{2} H_{\mathrm{P}}+m^{2} c^{4}-\zeta}=\frac{1}{2 m c^{2}} G_{\mathrm{P}}\left(\frac{\zeta}{2 m c^{2}}-\frac{m c^{2}}{2}\right),
$$

where $G_{\mathrm{P}}(\epsilon):=\left(H_{\mathrm{P}}-\epsilon\right)^{-1}$ is the resolvent of the non-relativistic Pauli Hamiltonian. In terms of this Pauli Green's function and the spin projection operator $A$, the Dirac Green's function can be put into the form

$$
G(z)=\frac{1}{2 m c^{2}}\left(\begin{array}{cc}
z+m c^{2} & A \\
A & z-m c^{2}
\end{array}\right) G_{\mathrm{P}}\left(\frac{z^{2}}{2 m c^{2}}-\frac{m c^{2}}{2}\right) .
$$

Some explicit examples have been worked out in ref. [32].

\subsection{The Resolvent of a Vector Boson in a Magnetic Field}

From Section 4.3, let us recall the relevant operators as follows:

$$
M_{ \pm}=H_{\mathrm{V}}+m c^{2}, \quad H_{ \pm}=H_{\mathrm{V}}^{2} / 2 m c^{2}, \quad A=\frac{\vec{\pi}^{2}}{2 m}-\frac{1}{m}(\vec{S} \cdot \vec{\pi})^{2},
$$


where the vector Hamilton $H_{\mathrm{V}}$ is given in Equation (44). Recalling that $A^{2}=H_{\mathrm{V}}^{2}$, we find for the iterated Green's functions

$$
g^{ \pm}(\zeta):=\frac{1}{\left(H_{\mathrm{V}}+m c^{2}\right)^{2}-H_{\mathrm{V}}^{2}-\zeta}=\frac{1}{2 m c^{2}} G_{\mathrm{V}}\left(\frac{\zeta}{2 m c^{2}}-\frac{m c^{2}}{2}\right)
$$

with $G_{\mathrm{V}}(\epsilon):=\left(H_{\mathrm{V}}-\epsilon\right)^{-1}$. The relativistic spin-one Hamiltonian explicitly reads

$$
H=\left(\begin{array}{cc}
m c^{2}+H_{\mathrm{V}} & A \\
-A & -m c^{2}-H_{\mathrm{V}}
\end{array}\right)
$$

and leads us to the Green's function

$$
G(z)=\frac{1}{2 m c^{2}}\left(\begin{array}{cc}
z+m c^{2}+H_{\mathrm{V}} & A \\
-A & z-m c^{2}-H_{\mathrm{V}}
\end{array}\right) G_{\mathrm{V}}\left(\frac{z^{2}}{2 m c^{2}}-\frac{m c^{2}}{2}\right) .
$$

\section{Summary and Outlook}

In this work we have considered relativistic one-particle Hamiltonians for an arbitrary but fixed spin $s$ and have shown that under the condition, that its even part commutes with its odd part, a SUSY structure can be established. Here, the SUSY transformations map states of negative energy to those of positive energy and vice versa. This is different to the usual SUSY concepts in quantum field theory where those charges transform bosonic into fermionic states and vice versa. As examples, we have chosen the physically most relevant cases of a massive charged particle in a magnetic field for the cases of a scalar particle $(s=0)$, a Dirac fermion $(s=1 / 2)$ and a vector boson $(s=1)$. In the case of a constant magnetic field, SUSY is broken for $s=0$ but remains unbroken for $s=1 / 2$ and $s=1$. The Witten index is only non-zero in the Dirac case but vanishes for the bosonic cases discussed. However, all three cases have resulted in the notable observation (28) that the FW-transformed Hamiltonian $H_{\mathrm{FW}}$ is entirely expressible in terms of a corresponding non-relativistic Hamiltonian $H_{\mathrm{NR}}$. As $H_{\mathrm{FW}}^{2}=H^{2}$, the relativistic energy-momentum relation can be put into the form

$$
H^{2}=m^{2} c^{4}+2 m c^{2} H_{\mathrm{NR}},
$$

which allows us to relate $H_{\mathrm{NR}}$ with the SUSY Hamiltonian (11).

There naturally arises the desire to also study the higher-spin cases $s \geq 3 / 2$. The corresponding free-particle Hamiltonians have been constructed, for example, by Guertin [46] in a unified way. However, as Guertin mentions, only for the cases discussed here, i.e., $s=0,1 / 2$ and 1 , the corresponding Hamiltonians are local operators.

Another route for further investigation would be to consider more exotic magnetic fields. For example, choosing an imaginary vector potential such that the kinetic momentum takes the form $\vec{\pi}=\vec{p}+\mathrm{i} m \omega \vec{r}$ in essence leads for $s=1 / 2$ to the so-called Dirac oscillator, which is know to exhibit such a SUSY structure [32]. To the best of our knowledge, the corresponding Klein-Gordon and vector boson oscillators have not yet been studied in the context of SUSY. Similarly, following the discussion of ref. [32] on the Dirac case, one may extend these discussion on a path-integral representation of the iterated Green's functions to the bosonic cases $s=0$ and $s=1$.

Funding: This research received no external funding.

Acknowledgments: The author has enjoyed enlightening discussions with Simone Warzel for which I am very thankful. The comments made by the referees are very much appreciated.

Conflicts of Interest: The author declares no conflict of interest. 


\section{Appendix A. Some Useful Relations for the Spin-One Case}

In this Appendix A we present a few relations which provide some additional steps used in Section 4.3. For an arbitrary magnetic field let us recall that the components of the kinetic momentum given by $\pi_{j}=p_{j}-(e / c) A_{j}$ obey the commutation relation

$$
\left[\pi_{k}, \pi_{l}\right]=(\mathrm{i} \hbar e / c) \varepsilon_{k l m} B_{m}
$$

where we use Einstein's summation convention for repeated indices. From this relation one may derive the commutator $\left[\pi_{k}, \vec{S} \cdot \vec{\pi}\right]=(\mathrm{i} \hbar e / c) \varepsilon_{k l m} S_{l} B_{m}$ which in turn leads us to

$$
\left[\vec{\pi}^{2}, \vec{S} \cdot \vec{\pi}\right]=(e \hbar / c)[\vec{S} \cdot \vec{B}, \vec{S} \cdot \vec{\pi}]+(e \hbar / c) S_{k} S_{l}\left(\pi_{l} B_{k}-B_{l} \pi_{k}\right) .
$$

For an arbitrary magnetic field the components of the kinetic momentum do not commute with the components of the magnetic field. However, if we now assume that the magnetic field is constant one may commute in the last term these components. That is, under the assumption that $\vec{B}=$ const. we arrive at

$$
\left[\vec{\pi}^{2}, \vec{S} \cdot \vec{\pi}\right]=(2 e \hbar / c)[\vec{S} \cdot \vec{B}, \vec{S} \cdot \vec{\pi}]
$$

which in turn results in

$$
\left[\vec{\pi}^{2},(\vec{S} \cdot \vec{\pi})^{2}\right]=\frac{2 e \hbar}{c}\left[(\vec{S} \cdot \vec{B}),(\vec{S} \cdot \vec{\pi})^{2}\right] .
$$

Note that relation (A3) was already given in Equation (3.17) of ref. [42]. With the help of (A4) it is easy to calculate the commutator

$$
\left[M_{ \pm}, A\right]=(g-2) \frac{e \hbar}{2 m^{2} c}\left[(\vec{S} \cdot \vec{B}),(\vec{S} \cdot \vec{\pi})^{2}\right]+(2 g-2) \frac{e \hbar}{2 m^{2} c}\left[\vec{\pi}^{2},(\vec{S} \cdot \vec{B})\right] .
$$

Noting that we have derived this under the assumption of a constant magnetic field the last commutator in above expression vanishes and hence we arrive at Equation (43). Note that $\left[S_{k}, S_{l}\right]=\mathrm{i} \varepsilon_{k l m} S_{m}$ and therefore the first term on the right-hand-side above even for a constant magnetic field only vanishes when $g=2$.

With the assumption that the magnetic field is constant and utilising below properties of the spin-one matrices

$$
S_{i} S_{j} S_{k}+S_{k} S_{j} S_{i}=\delta_{i j} S_{k}+\delta_{j k} S_{i}, \quad \varepsilon_{i j k} S_{i} S_{j} B_{k}=\mathrm{i} \vec{S} \cdot \vec{B}
$$

one may verify the relations (see Equations (3.18) and (3.19) in ref. [42])

$$
\begin{aligned}
& (\vec{S} \cdot \vec{\pi})^{4}=\left(\vec{\pi}^{2}-\frac{2 e \hbar}{c}(\vec{S} \cdot \vec{B})\right)(\vec{S} \cdot \vec{\pi})^{2}+\frac{e \hbar}{c}(\vec{B} \cdot \vec{\pi})(\vec{S} \cdot \vec{\pi}), \\
& \left\{(\vec{S} \cdot \vec{B}),(\vec{S} \cdot \vec{\pi})^{2}\right\}=\left(\vec{\pi}^{2}-\frac{e \hbar}{c}(\vec{S} \cdot \vec{B})\right)(\vec{S} \cdot \vec{B})+(\vec{B} \cdot \vec{\pi})(\vec{S} \cdot \vec{\pi}) .
\end{aligned}
$$

Noting that for $g=2$ we have

$$
H_{\mathrm{V}}:=\frac{\vec{\pi}^{2}}{2 m}-\frac{e \hbar}{m c}(\vec{S} \cdot \vec{B}), \quad A=\frac{\vec{\pi}^{2}}{2 m}-\frac{1}{m}(\vec{S} \cdot \vec{\pi})^{2}
$$

and with above relations (A7) immediately follows that $A^{2}=H_{\mathrm{V}}^{2}$ as claimed in the main text. Finally, let us mention the explicit form of the energy eigenfunctions can also be found in ref. [42].

\section{References}

1. Klein, O. Quantentheorie und fünfdimensionale Relativitätstheorie. Z. Phys. 1926, 37, 895-906. [CrossRef]

2. Gordon, W. Der Comptoneffekt nach der Schrödingerschen Theorie. Z. Phys. 1926, 40, 117-133. [CrossRef]

3. Dirac, P.A.M. The Quantum Theory of the Electron. Proc. R. Soc. A 1928, 117, 610-624. 
4. Dirac, P.A.M. The Quantum Theory of the Electron Part II. Proc. R. Soc. A 1928, 118, 351-361.

5. Proca, A. Sur la théorie ondulatoire des électrons positifs et négatifs. J. Phys. Radium 1936, 7, $347-353$. [CrossRef]

6. Dirac, P.A.M. Relativistic Wave Equations. Proc. R. Soc. A 1936, 155, 447-459.

7. Fierz, M. Über die relativistische Theorie kräftefreier Teilchen mit beliebigem Spin. Helv. Phys. Acta 1939, 12, 3-37.

8. Fierz, M.; Pauli, W. On Relativistic Wave Equations for Particles of Arbitrary Spin in an Electromagnetic Field. Proc. R. Soc. A 1939, 173, 211-232.

9. Bhabha, H.J. Relativistic wave equations for the elementary particles. Rev. Mod. Phys. 1945, 17, $200-216$. [CrossRef]

10. Bargmann, V.; Wigner, E.P. Group Theoretical Discussion of Relativistic Wave Equations. Proc. Natl. Acad. Sci. USA 1948, 34, 211-223. [CrossRef]

11. Foldy, L.L.; Wouthuysen, S.A. On the Dirac Theory of Spin 1/2 Particles and Its Non-Relativistic Limit. Phys. Rev. 1950, 78, 29-36. [CrossRef]

12. Foldy, L.L. Synthesis of Covariant Particle Equations. Phys. Rev. 1956, 102, 568-581. [CrossRef]

13. Feshbach, H.; Villars, F. Elementary Relativistic Wave Mechanics of Spin 0 and Spin 1/2 Particles. Rev. Mod. Phys. 1958, 30, 24-45. [CrossRef]

14. Duffin, R.J. On The Characteristic Matrices of Covariant Systems. Phys. Rev. 1938, 54, 1114. [CrossRef]

15. Kemmer, N. The particle aspect of meson theory. Proc. R. Soc. A 1939, 173, 91-116.

16. Yukawa, H.; Sakata, S.; Taketani, M. On the Interaction of Elementary Particles. III. Proc. Phys.-Math. Soc. Jpn. 1938, 20, 319-340. [CrossRef]

17. Sakata, S.; Taketani, M. On the Wave Equation of Meson. Proc. Phys.-Math. Soc. Jpn. 1940, 22, 757-770.

18. Corben, H.C.; Schwinger, J. The Electromagnetic Properties of Mesotrons. Phys. Rev. 1940, 58, $953-968$. [CrossRef]

19. Schrödinger, E. The wave equation for spin 1 in Hamiltonian form. Proc. R. Soc. A 1955, 229, $39-43$.

20. Silenko, A. Exact form of the exponential Foldy-Wouthuysen transformation operator for an arbitrary-spin particle. Phys. Rev. A 2016, 94, 032104. [CrossRef]

21. Simulik, V.M. Relativistic Equations for arbitray Spin, especially for the Spin $s=2$. Ukr. J. Phys. 2019, 64, 1064-1068. [CrossRef]

22. Nicolai, H. Supersymmetry and spin systems. J. Phys. A 1976, 9, 1497-1506. [CrossRef]

23. Witten, E. Dynamical breaking of supersymmetry. Nucl. Phys. B 1981, 188 513-554. [CrossRef]

24. Cooper, F.; Khare, A.; Sukhatme, U. Supersymmetry and quantum mechanics. Phys. Rep. 1995, 251, $267-385$. [CrossRef]

25. Junker, G. Supersymmetric Methods in Quantum and Statistical Physics, 1st ed.; Springer: Berlin, Germany, 1996.

26. Jackiw, R. Fractional charge and zero modes for planar systems in a magnetic field. Phys. Rev. D 1984, 29, 2375-2377. [CrossRef]

27. Ui, H. Supersymmetric quantum mechanics and fermion in a gauge field of $(1+2)$ dimensions. Prog. Theor. Phys. 1984, 72, 192-193. [CrossRef]

28. Sarma, S.D.; Adam, S.; Hwang, E.H.; Rossi, E. Electronic transport in two-dimensional graphene. Rev. Mod. Phys. 2011, 83,407-470. [CrossRef]

29. Thaller, B. The Dirac Equation; Springer: Berlin, Germany, 1992.

30. Znoil, M. Relativistic supersymmetric quantum mechanics based on Klein-Gordon equation. J. Phys. A 2004, 37, 9557. [CrossRef]

31. Junker, G. Supersymmetric Methods in Quantum, Statistical and Solid State Physics, Enlarged and Revised Edition; IOP Publishing: Bristol, UK, 2019.

32. Junker, G.; Inomata, A. Path integral and spectral representations for supersymmetric Dirac-Hamiltonians. J. Math. Phys. 2018, 59, 052301. [CrossRef]

33. Witten, E. Constraints on supersymmetry breaking. Nucl. Phys. B 1982, 202, 253-316. [CrossRef]

34. Fock, V. Bemerkung zur Quantelung des harmonischen Oszillators im Magnetfeld. Z. Phys. 1928, 47, 446-448. [CrossRef]

35. Landau, L. Diamagnetismus der Metalle. Z. Phys. 1930, 64, 629-637. [CrossRef]

36. Aharonov, Y.; Casher, A. Ground state of a spin- $\frac{1}{2}$ charged particle in a two-dimensional magnetic field. Phys. Rev. A 1978, 19, 2461-2462. [CrossRef] 
37. Rabi, I.I. Das freie Elektron im homogenen Magnetfeld nach der Diracschen Theorie. Z. Phys. 1928, 49, 507-511. [CrossRef]

38. Young, J.A.; Bludman, S.A. Electromagnetic Properties of a Charged Vector Meson. Phys. Rev. 1963, 131, 2326-2334. [CrossRef]

39. Krase, L.D.; Lu, P.; Good, R.H., Jr. Stationary States of a Spin-1 Particle in a Constant Magnetic Field. Phys. Rev. D 1971, 3, 1275-12798. [CrossRef]

40. Tsai, W.-Y.; Yildiz, A. Motion of Charged Particles in a Homogeneous Magnetic Field. Phys. Rev. D 1971 4, 3643-3648. [CrossRef]

41. Goldman, T.; Tsai, W.-Y. Motion of Charged Particles in a Homogeneous Magnetic Field II. Phys. Rev. D 1971, 4, 3648-3651. [CrossRef]

42. Daicic, J.; Frankel, N.E. Relativistic spin-1 bosons in a magnetic field. J. Phys. A Math. Gen. 1993, 26, 1397-1408. [CrossRef]

43. Silenko, A.J. High precision description and new properties of a spin-1 particle in a magnetic field. Phys. Rev. D 2014, 89, 121701. [CrossRef]

44. Junker, G. Supersymmetric quantum mechanics requires $g=2$ for vector bosons. Eur. Phys. Lett. 2020, 130, 30003. [CrossRef]

45. Weaver, D.L. Application of a single-particle-theory calculation of $g-2$ to spin one. Phys. Rev. D 1976, 14, 2824-2825. [CrossRef]

46. Guertin, R.F. Relativistic Hamiltonian Equations for Any Spin. Ann. Phys. 1974, 88, 504-553. [CrossRef]

(c) 2020 by the author. Licensee MDPI, Basel, Switzerland. This article is an open access article distributed under the terms and conditions of the Creative Commons Attribution (CC BY) license (http:/ / creativecommons.org/licenses/by/4.0/). 